\title{
Knowledge Management in PhD Students' Information Seeking Behaviors
}

\author{
Yi Zhou a, Siyi Chen ${ }^{b}$, Mengshi Xu ${ }^{c}$ \\ Nanyang Technological University, Singapore \\ aw170016@e.ntu.edu.sg, bchen1173@e.ntu.edu.sg, 'cmxu014@e.ntu.edu.sg
}

\begin{abstract}
This article discusses how to apply knowledge management strategy to PhD students' academic information seeking behavior. It reviews the definitions and relations of information and knowledge, personal information management and personal knowledge management, and knowledge management and information seeking. Based on the knowledge management perspective in information seeking behavior, the interviews are conducted. Thirty PhD students participate in the interview and a systematic analysis is shown to readers. Based on the results of the interviews, authors focus on the barriers which theses PhD students are faced in their academic research. To address the problems, this article provides three practical recommendations according to the main problems which the students have. All the recommendations are under the knowledge management perspective and are aimed to connecting knowledge management strategies with PhD students' information seeking behavior. These knowledge management suggestions can enhance their research and improve the outcomes of the research in an efficient way.
\end{abstract}

Keywords: Knowledge management, information seeking behavior, academic research, knowledge management practices.

\section{Introduction}

It is known that $\mathrm{PhD}$ students have heavy research tasks, and they need to do a large amount of academic information seeking work. The study of information seeking behavior of researchers can be dated back to the late 1940s. Since that time, a great number of studies have been carried out on various aspects of the information seeking behavior of researchers. However, there are many difficulties in information seeking for research students. Information seeking is a process of construction that begins with uncertainty [1] and it is a critical and fundamental step for $\mathrm{PhD}$ students to form a necessary basis on their academic papers. In this case, the process of information seeking and essay writing are continuous to complete the whole research work. PhD students are still in the study phase so that they may need guidance from experienced experts and communication with others, but in reality, they may lack this kind of channels to get help. Besides, it is may be hard for doctoral candidates to find the valuable resources which can be transferred to systematic information that can be contributed to the dissertations. In addition, $\mathrm{PhD}$ students may encounter too much information and do not know how to organize it well. Under this situation, the wide variation in information seeking behavior implies a need for further categorization of information need and information sources [2].

Information and knowledge are linked closely. At the same time, the encouragement of $\mathrm{PhD}$ students to adopt the better principles and practice of information seeking should not take place in isolation from knowledge management strategies [2]. We believe there is of great value to $\mathrm{PhD}$ students that develop knowledge management skills to achieve their research objectives [3]. After reviewing a considerable number of literature and inspired by what we learned in our current major as well, we wish to help contribute towards connecting the knowledge management with information seeking to address the concerns exist regarding the gaps in knowledge base of the research reports. We attempt to interview $30 \mathrm{PhD}$ students to investigate their information seeking behavior when they work on academic research. Then we will do a deep analysis on their information seeking process using knowledge management perspective. More specifically, we need to find the concrete gaps between their ideal needs and real situation of information seeking. Besides, we need to explore the different sources use among multidisciplinary $\mathrm{PhD}$ students and explore the problems existing in their 
information seeking behaviour to help them apply proper knowledge management practices in the process of information seeking.

After the analysis of the result of interviews, we are dedicated to exploring the practical ways in helping doctorates to acquire "valued knowledge" which can further help them enhance the understanding of their research topics and generate papers of good quality. We tend to help $\mathrm{PhD}$ students identify the key features of the knowledge management practices which we recommend for them. Through this way, effective knowledge management can help $\mathrm{PhD}$ students spur innovation, improve the channels of valuable sources of information, and achieve operational techniques to manage information [3].

\section{Methodology}

Methodologically, this study was a descriptive research because we have no control over any variables. The descriptive research was also called Ex post factor research which is a habitual term used by researchers in social science studies [4]. Given that this article was focused on describing what has happened and what is happening, we adopted the semi-structured interviews.

This article aimed to describe the currently existing phenomenon of information seeking behavior for doctoral candidates and postdoctoral staff in their daily research. After uncovering the investigated results, the commonalities and differentiation among participants from different disciplines would be analyzed.

\subsection{Preliminary Works}

\subsubsection{Method of Data Collection}

Primary data was collected originally from the interviewees, which could reflect the attitude and behavior of them more precisely. The interview method for data collection was determined after the research objectives had been decided. On the basis of the objectives, we thought that semi-structured interviews were the right way to conduct our study,

The advantage of adopting a semi-structured interview was that the time allotted is set up ahead, while in "unstructured interviews", there was no time limit and it is hard to seperate the unimportant parts from the significant ones. In addition, compared to semi-structured approach, the totally structured interviews may not be able to identify the true feelings of students

\subsection{Sampling Method}

Our study primarily was focused on doctoral candidates and postdoctoral who were from Electrical and Electronic Engineering, School of Computer Science and Engineering, School of Civil and Environmental Engineering, School of Materials Science and Engineering, School of Mechanical and Aerospace Engineering, School of Physical and Mathematical Sciences, School of Social Sciences, Nanyang Business School, and Interdisciplinary Graduate School of NTU. We recruited $30 \mathrm{PhD}$ students in total through personal network, social network (e.g. Wechat group, Facebook, Whatsapp and approach them in academic sharing events, etc.). From these schools, covered multidisciplinary fields including Engineering, Science, Social Science, and Business. The similarities and differences of information seeking behavior among these participants would be analyzed.

\section{Research Findings}

\subsection{Information Seeking Behavior Analysis}

The information seeking behaviour which we tended to analyze can be divided into three parts, which are the ways to organize information, whether to ask for help from experts and whether to attend relevant meetings and cross-field events. 


\subsubsection{Ways to Organize Information}

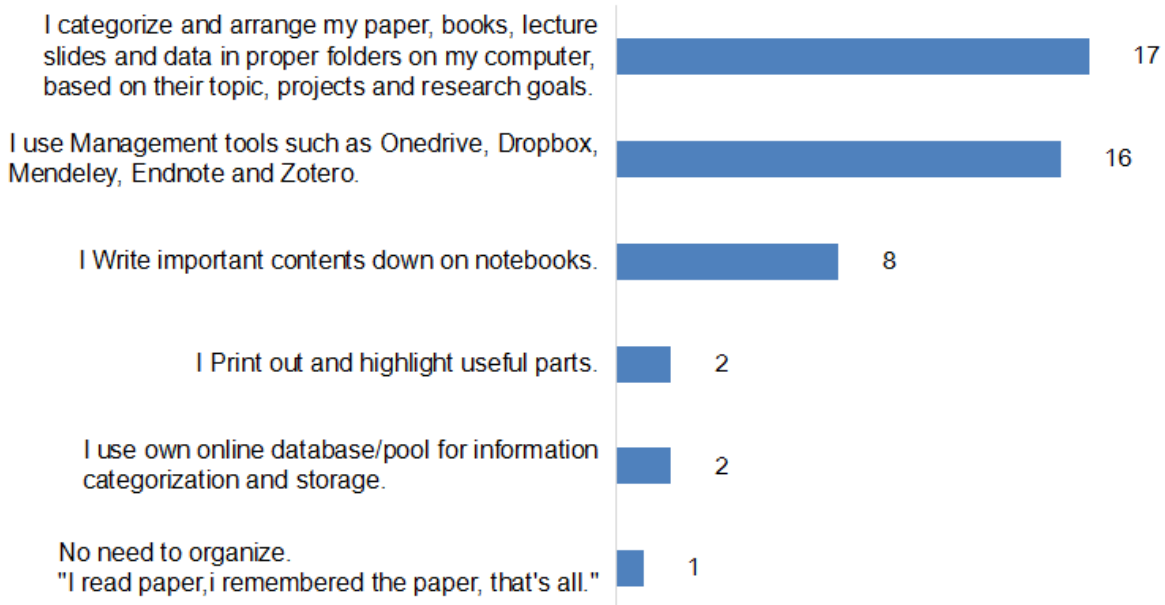

Figure 1. The ways to organize information.

When asking the participants how to organize the information they sought to be used in their research, the majority of participants came up with the ideas of using management tools (software like Endnote, Mendeley and OneDrive are among the lists of popular research tools), as well as manually categorizing various kinds of references according to their projects and research goals. Around $70 \%$ of interviewees who worked on the research topics in social science field claim that they often wrote notes down on their notebooks, sometimes they printed the references out and highlighted the interested parts. One interviewees from school of SPMS shows his indifference to information organization, as he trusts his excellent memory.

In this case, we found that there is similarity existed in the common management tools which we mentioned in the last paragraph.

\subsubsection{Whether to Ask for Help from Experts}

When talking about whether they would seek outside help like asking their mentors or other experts for help, eighteen of thirty $\mathrm{PhD}$ students answered that they asked their mentors or collaborators for seeking information. Because their mentors or collaborators were more experienced and can gave some guidance on their research topics. For example, a student from EEE said that his supervisor always delivered the important references such as papers, books as well as the references his supervisor had read before. Supervisors can suggest directions and help students get away from being lost. Also, they would ask their collaborators for valuable information in discussion, they can provide more details and share the appropriate methodologies. It's quite useful since it can save a lot of time.

However, there are still 7 students who didn't ask for help, some of them preferred to do it alone, some said because their research areas were very unique, and even though their supervisors couldn't understand or resolve that.

\subsubsection{Whether to Attend Relevant Meetings and Cross-field Events}

This question is asked by "Do you usually attend the meetings or sessions that are related to the research in your field?" and "Will you consider attending other cross-field events to get access to information? If yes, do you think how does it help? If not, why?" As for attending meetings in the field of study, 25 students do attend the meetings or sessions that are related to their research fields. For them, group meetings and annual conferences are mandatory. Others would attend if those seminars or sessions attract them. Most of them felt attending the sessions that were related were super useful. For example, one $\mathrm{PhD}$ student said "They gave a wide picture of state-of-art approaches used in areas similar to mine. And I used them to get inspired about my own research." Another PhD student from SPMS said that he could get some new ideas and consult current problems with others 
who also attended the meetings or seminars. For the students who attended regional or international conferences, they could exchange information with peers.

On the other hand, 24 students tend to consider attending cross-field events to gain knowledge. Some of them thought that it would broaden their views and provide perspective points. One PhD student from EEE said, "Because most of the things happening in today's world are involved in various fields. Your basic understanding after attending a cross-field event may become important or relevant in near future." Another student said, "Since I am dealing with engineering and applied science, new field is always welcome. My projects are inspired by natural processes. We never know where new ideas are going to appear." While some attend just for fun or out of curiosity. Because interdisciplinary knowledge can provide a new way of thinking. A special case is that a student whose major itself is cross-disciplinary and he is from Interdisciplinary Graduate School. Many other students would not consider attending cross-field events. Some claim they are not interested in such social conditions and others may think it is really a waste of time.

\subsection{Barriers and Needs Analysis}

\subsubsection{Barriers}

Then we focus on the barriers and main needs of these respondents. It is shown clearly that 11 participants are plagued by the sources of knowledge. Firstly, some of them need to learn a variety of knowledge except his major. Nowadays the standards for research are improving, thus researchers are required to know multidisciplinary knowledge so that they can seek more valuable materials purposefully and then make the in-depth and extensive researches. It consumes a lot of time for them to build up the basics of other subjects. In addition, sometimes there is not so much publications or papers refer to the topics they are prepared to study, or sometimes the authors do not put all the information obtained from their researches or skip some important parts. In this case, these participants seem to feel confused and may be stuck in their information seeking process. As for some science and engineering interviewees, getting the same result as stated in previous articles is hard enough so that they cannot proceed to the following information-seeking step on the basis of repeating experiment results.

Besides, there are also 6 participants are struggling with no creativity or novelty It is necessary to have a general research direction before seeking the information they need to use. Many high - level debates on 'how to' be published portray an important role in novelty. Journals (and their reviewers) encourage originality and single out novelty as a major criterion for assessing the value of a contribution [5]. As a result, novelty is one of the important criteria to decide whether the paper is of high quality. However, it is obvious that people cannot always be full of creativity and it is actually very hard to have new ideas and inspirations, so participants may be anxious about how to make their work more valuable and how to refine their frameworks or models so that they are very likely to feel pressure to "compete" with others. The issues of limited knowledge we mentioned before may lead to limited innovative ideas as well.

In addition, some participants complain to us that they lack supervising by experienced instructors who have the same background as them. They cannot receive enough guidance from others and have no one to discuss about the ideas and problems they encounter in their research. For example, when they need to decide whether to proceed with further information seeking actions when initial outcomes are not as positive as planned, or there are so many destinations they want to go before searching the information. If senior and experienced experts appear at this time and give suggestions, they can make the process of students' research much smoother.

\subsubsection{Needs}

To better understand participants concerns, we also analyze the areas in which they want to seek help. Their needs can be divided into two main parts. One is about how to manage knowledge in an efficient and appropriate way in the process of information seeking, the other is about how to embrace new resources and connect cross-disciplinary knowledge. They also care about how to get help from experts and seniors. 
Therefore, it can be seen that the participants' needs are consistent with the barriers they have claimed before. At the same time, the result of their demands highlights the importance of three issues: no expert who can go for help, problems of finding valuable information and lack of creativity and novelty.

\section{Discussion and Suggestion}

On the basis of our interview results which are analyzed before, we find that it is a good way to introduce knowledge management strategy in their information seeking process because the implementation of knowledge management practices can make them seek useful information both efficiently and effectively.

As a result, to address the main three barriers students encounter, here we propose several suggestions of knowledge management practices: building the expert assoc,iation as "brain trust" to provide constructive and professional advice for students, encouraging students to participant in events and building the communities and implementing technologies for PKM to facilitate knowledge reuse and creation.

\subsection{Building the Expert Association}

One of the big problems is that when PhD students want to seek for help, there are no experts around, especially when the deadline is coming. Under this urgent situation, students tend to feel anxious.

Here we suggest on building expert associations in school. The structure of the expert association can be seen as below.

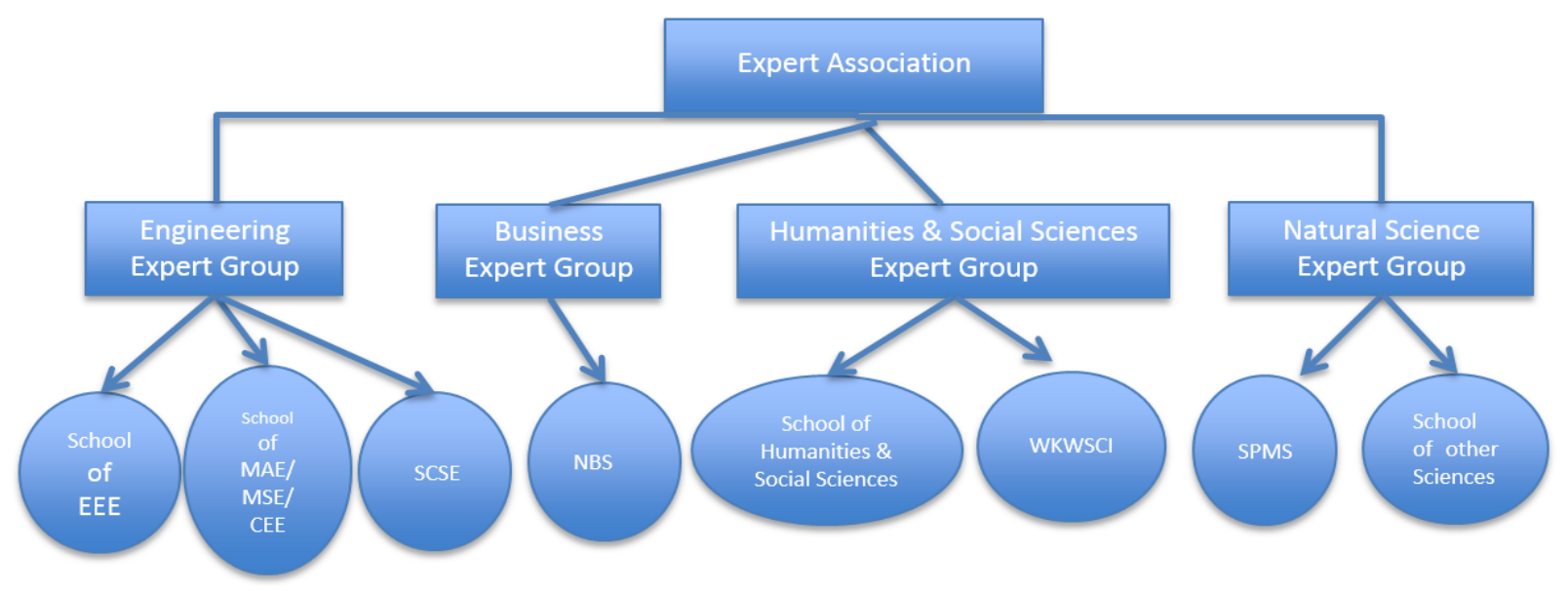

Figure 2. The structure of the expert association

Firstly, we can establish an expert group at each academy, and then invite associate professors or senior lecturers who are experienced in each study field as an expert, and then show their available time according to their own arrangements. A timetable will be made, such as Expert A is available on Tuesday and Thursday afternoon, and the information is published on school official website, it can be noticed by email with detailed information, students can seek the subject expert according to their current needs within the domain.

Then we can build up an expert association on campus to bring together all the experts from different academies. Since some research projects are cross some fileds, and there is a need to set up a community to facilitate the communication of experts among different fields, thus to facilitate knowledge sharing and knowledge reuse. And the timetable and profile of experts will be published and updated timely on the official website. The profile includes the expert's basic information, professional experience, research achievements, study domain, personal style, and so on. If students need to ask questions about another fields which they do not understand and cannot get effective and valuable information after searching from internet or papers, then they can turn to experts who are at 
school. Students just need to make an appointment based on the time slot. So that they will get satisfied answers to their problems.

The benefit to build expert associations is to eliminate or mitigate the risks that when students need help, the professors or supervisors are not at office, and sometimes they cannot get timely response after emailing their questions. In building up this expert association in school, it will be much easier for students to seek help in a timely manner, therefore they will feel certain about the things and be less anxious.

\subsection{Participating in Events and Building Communities}

In the process of academic information seeking, creativity is a critical factor to determine the direction and topic of information which $\mathrm{PhD}$ students are going to collect. As a result, it is very significant for them to be inspired to generate new ideas. In the previous analysis of interview results, we can see that students from applied discipline such as finance and accounting in business school are more likely to attend some academic events even though they are may be cross-disciplinary. But this kind of students make up a small part of all respondents and quite a number of students are not willing to attend those events because they think it is a waste of time.

However, attending academic events is an effective and feasible way to make students generate creative ideas. In this case, attendees can communicate with each other and students can always learn something from others during the process of interacting with others. It can not only widen your eyes, but also can acquire new knowledge, new experience by meeting new people. They may encounter some problems and challenges which need to be addressed as well. Therefore, learning can occur when the learners solve problems and make decisions. As we all know, learning can stimulate creativity so in this way, it is relatively possible for students to come up with creative ideas.

On the basis of the recognition of the usefulness of cross-field events. We can build up a community which gathers researchers from various domains. This community is made up of different forums and each of them has their own theme. It refers to a social network of learning and it is initially a practice of individual learning. The establishment of the community reveals that creation and innovation occur through informal and situated social interactions, rather than just seeking information in a closed environment. Under this situation, people who are involved in the community can regularly share professional resources such as knowledge, experience, tools and solutions. In addition, they can interact actively and learn from one another.

Creative and innovative ideas may be generated by observation because students can learn by observing how other people from the same background interpret their ideas and solve the problems. More importantly, students can also obtain the outcome of learning by better participation when they learn new things by directly engage in the aim of making decision in choosing a creative research topic and then doing deep research.

However, what is worth mentioning is that too many social network events are likely to cause useless social networking. It can make students waste too much time and unable to concentrate on following process of information seeking and research study. To avoid this situation, students need to know exactly the theme of the activities and have a preliminary judgment about the usefulness of these events. Speaking of the community, we can designate personnel to supervise the quality of information transferred in each forum, staff can also play a role in steering the communication and making everything get along well.

\subsection{Implementing Technologies for Personal Knowledge Management}

Generally, PKM (Personal Knowledge Management) is concentrated on facilitating individuals to learn, work and socialize more efficiently. The primary perspective of PKM is to help people manage their knowledge processes (i.e. knowledge collection, codification and storage of knowledge, knowledge reuse and knowledge exchanges with others) [6]. In order to enable researchers to manage their knowledge better, this article will put forward several practical knowledge management technologies for them to improve the working efficiency. These technologies involve in effective IR 
(Information Retrieval) methods, methods of knowledge organization and popular Web 2.0 technologies for personal knowledge diffusion.

\subsubsection{IR Methods}

Some interviewees respond that it is hard to seek for useful information which can be used in their study, or other interviewees claim that it takes a lot of time to search information in order to avoid duplicated research. The main reason is that research students have a lack of knowledge of IR methods. Most of students use keywords to search journal articles in Google scholar and NTU library. Therefore, our suggestion is that school should offer specialized general courses for research students to train their IR techniques. For example, using Advanced Information Retrieval to instead simple keywords retrieval is a better choice because most of search engines currently apply Boolean Retrieval model for their search function. By investigating, few researchers know or use this kind of channel.

Advanced Information Retrieval mainly use Boolean retrieval model (using Boolean elements, e.g. AND, NOT, and OR) but is not limited to this. Users can change for another search mode and expander, and they can also select an optional field. Designers of search engines may use metadata (e.g. author, publication date, language and location), which is extracted from resources for users to limit their search results. Furthermore, school should encourage students to use Database Retrieval. Most of students may be confused with numerous various databases. Therefore, we suggest schools train research students to use such databases related to their fields.

\subsubsection{Organization of Knowledge}

Researchers search a large quantity of information which will be used in their research, many of them have limited idea about how to organize knowledge they obtained from different channels. Take an example of searched articles, about over half of interviewees use different folders to allocate them. With the great increase of documents or other resources, it becomes harder and harder to tell which documents in which folders. In order to cope with this issue, we suggest methodology of classification (i.e. ontology and taxonomy) for researchers to build their own knowledge repository (refers to the Figure 3. below).

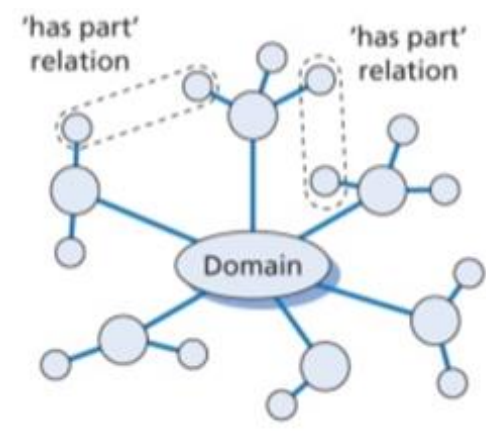

ONTOLOGY

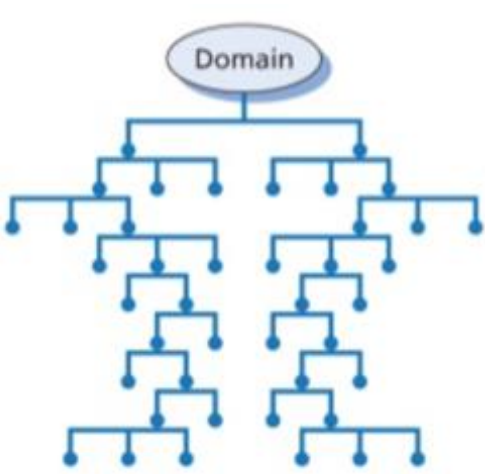

TAXONOMY

- represent different ideas and concepts

Figure 3. The taxonomy and ontology in KM (Jashapara, 2004).

As the above figure shows, taxonomy is a method of classification, which can be used to categorize knowledge based on nature of concepts. At length, taxonomy can represent the hierarchical relationship among entities. According to these categorizations, researcher could rapidly position or location the related knowledge. Ontology is a more complex application for classification, which involves both entities and their complicated relationships such as 'is-a', 'part-of' and so forth. Ontology also can interweave the human understanding of concepts and ideas with the resources they obtained for knowledge sharing and re-use [2]. 
Furthermore, researchers respond that they spend a substantial amount of time to browse and read articles to find out how they are related to each other and where they fall into the problem domain. Ontology can help people construct sematic knowledge network. Many tools (i.e. ontology languages) for us to choose from are practically useful (e.g. QuizRDF, XML and DAML+ OIL). Therefore, ontology could be a useful tool for researchers to organize knowledge and to stimulate them to create new ideas.

\section{Implications}

This paper can be seen as a descriptive analysis study on information seeking behavior of $\mathrm{PhD}$ students from knowledge management perspective. The semi-structured interview and descriptive analysis method has been tested quite useful in shaping our view.

In addition, the focus point of this paper is very practical, which is based on the process of PhD students' academic research. This process is embedded in their daily study and the research problems we designed in the interview is all about PhD students' information seeking at their normal research work. Based on the barriers we sum up in the interview, the knowledge management practices we come up with tend to be feasible and practical as well. We conduct the careful planning to put forward the approaches of information seeking to enable $\mathrm{PhD}$ students to keep up to date and improve the quality of their research. At the same time, the practices we offer can be applied by both individuals and education institutions since these approaches have wide scope of applications and are easy to operate.

\section{Limitations \& Recommendations}

This paper is a preliminary analysis based on the inquiry results. Due to time limit, and the restrictions on the number of participants, the participants may not represent all the researchers. And because of the particularity of their research directions, the comparison of information seeking behavior among researchers from different disciplines is not that obvious as expected. Thus, further study based on a more detailed qualitative analysis is required.

On the other hand, there exists a problem that different people have different personalities, especially when participants are from different cultural background. For example, when we conduct the interviews, most Russian participants prefer to write answers instead of taking face-to-face interviews, and so as some Chinese participants do. In this case, we provide e-mail communication choice when we invite participants to take part in our investigation. Therefore, when facing cultural issues or personality difference, it is suggested that we show respect and adopt alternatives to avoid this kind of risk.

\section{Conclusion}

This study conducts the interview to explore $\mathrm{PhD}$ students' information seeking behaviour. In this case, this article develops and applies knowledge management perspectives to the information seeking behaviour. Through this kind of combination between knowledge management and information seeking, there are several knowledge management practices put forward to help students conduct the process of information seeking more effectively and efficiently, which can make them generate the academic papers of higher quality.

Although knowledge management is very important, and many people have heard the term, not many of them really know what it is and how it will help in the real world. In the future, we think education institutions such as universities and libraries may organize the knowledge management workshops, which can bring a series of knowledge management theory and practices to the audience. In this way, knowledge management can be popularized, and people can connect with each other by knowledge sharing. It will benefit the whole society in the long term. 


\section{References}

[1]. Kuhlthau, C. C. (1991). Inside the search process: Information seeking from the user's perspective. Journal of the American society for information science, 42(5), 361.

[2]. Dawes, M., \& Sampson, U. (2003). Knowledge management in clinical practice: a systematic review of information seeking behavior in physicians. International journal of medical informatics, 71(1), 9-15.

[3]. Kidwell, J. J., Vander, K., Johnson, S. L., \& Sandra, L. (2000). Knowledge Management Practices Applying Corporate in Higher Education. Educause Quarterly, (4), 28-33.

[4]. Kothari, C. R. (2004). Research methodology: Methods and techniques. New Age International.

[5]. Patriotta, G. (2017). Crafting papers for publication: Novelty and convention in academic writing. Journal of Management Studies, 54(5), 747-759.

[6]. Razmerita, L., Kirchner, K., \& Sudzina, F. (2009). Personal knowledge management: The role of Web 2.0 tools for managing knowledge at individual and organisational levels. Online information review, 33(6), 1021-1039.

[7]. Liyana, S., \& Noorhidawati, A. (2017). How graduate students seek for information: Convenience or guaranteed result?. Malaysian Journal of Library \& Information Science, 19(2).

[8]. Alavi, M., \& Leidner, D. E. (2001). Knowledge management and knowledge management systems: Conceptual foundations and research issues. MIS quarterly, 107-136.

[9]. Barrett, A. (2005). The Information-Seeking Habits of Graduate Student Researchers in the Humanities1. The Journal of Academic Librarianship, 31(4), 324-331.

[10]. Belkin, N. J. (1984). Cognitive models and information transfer. Social Science Information Studies, 4(2-3), 111-129.

[11]. Ellis, D., Cox, D., \& Hall, K. (1993). A comparison of the information seeking patterns of researchers in the physical and social sciences. Journal of documentation, 49(4), 356-369.

[12]. Weiler, A. (2005). Information-seeking behavior in generation Y students: Motivation, critical thinking, and learning theory. The journal of academic librarianship, 31(1), 46-53.

[13]. Liyana, S., \& Noorhidawati, A. (2017). How graduate students seek for information: Convenience or guaranteed result?. Malaysian Journal of Library \& Information Science, 19(2).

[14]. Mai, J. E. (2016). Looking for information: A survey of research on information seeking, needs, and behavior. Emerald Group Publishing.

[15]. Mai, J. E. (2016). Looking for information: A survey of research on information seeking, needs, and behavior. Emerald Group Publishing. 